\title{
MATERIALS FOR COMPACT THERMAL ENERGY STORAGE: A NEW IEA JOINT SHC/ECES TASK
}

\author{
Marco Bakker, Wim G.J. van Helden \\ Energy Research Centre of the Netherlands (ECN) \\ P.O. Box 1, 1755 ZG Petten, The Netherlands \\ Andreas Hauer \\ Zentrum für Angewandte Energieforschung (ZAE) \\ Walther-Meissner-Strasse 6, D-85748 Garching, Germany
}

\begin{abstract}
A new IEA Task has recently been initiated to develop new storage materials. This Task is implemented as a Joint Task between the Solar Heating and Cooling (SHC) and Energy Conservation through Energy Storage (ECES) Implementing Agreements, and is entitled "IEA SHC/ECES 42/24: Compact thermal energy storage: material development for system integration".

The objective of this Task is to develop advanced materials for compact storage systems, suitable not only for solar thermal systems, but also for other renewable heating and cooling applications such as solar cooling, micro-cogeneration, biomass, or heat pumps. The Task will cover phase change materials, thermochemical and sorption materials, and composite materials and nanostructures, and will include activities such as material development, analysis, and engineering, numerical modelling of materials and systems, development of storage components and systems, and development of standards and test methods.

The main added value of this Task is to combine the knowledge of experts from materials science as well as solar/renewable heating and energy conservation. The Task has officially started on January 1, 2009, and will last for four years.
\end{abstract}

\section{BACKGROUND}

Thermal energy storage is an important technology for renewable energy systems. By improving the effectiveness of thermal storage, the effectiveness of all renewable energy technologies that supply heat can be improved.
Particularly for solar thermal systems, thermal energy storage is essential. To reach high solar fractions, it is necessary to store heat (or cold) efficiently for longer periods of time. Until now, no cost-effective compact storage technologies are available to do this. For high solar fraction systems, hot water stores are expensive and require very large volumes of space. Alternative storage technologies, such as phase change materials (PCMs) and thermochemical materials (TCMs) are available on a laboratory scale. However, more research and development is needed before these technologies can be developed into commercial solutions.

In several IEA Annexes, both ongoing and completed ${ }^{1}$, it was concluded that materials are the main bottleneck for finding effective solutions for compact thermal energy storage, and that there is a need for new storage materials with a higher specific energy storage density and lower material cost.

Around the world, several groups are working on either thermal energy storage materials or applications. However, these activities are not sufficiently linked. The current activities are either limited to specific applications, or to specific materials. What is needed is a way to bring the ongoing work on materials and applications together. This is one of the most important motivations behind the new Joint IEA SHC/ECES Task/Annex 42/24, that has started officially on January $1,2009$.

After two expert meetings, one in October 2007 in Zürich, Switzerland, and another in April 2008 in Petten,

\footnotetext{
${ }^{1}$ Most notably IEA ECES Annex 17 [1] and IEA SHC Task 32 [2].
} 
the Netherlands, a proposal for the Task was presented to both the ECES and SHC ExCos in November and December 2007, respectively. Both ExCos were very positive on the scope and topic of this task, and have approved the start of this Task as of January 1, 2009. The task will last four years, i.e. until December 2012. The main challenge for this task is to bring together material experts and application experts (particularly solar applications, given the primary scope of the task). Because of this, it was decided that this task should take the form of a joint task between these two Implementing Agreements.

The Task's will be coordinated by two Operating Agents: Wim van Helden of ECN, The Netherlands, and Andreas Hauer of ZAE Bayern, Germany. The official kick-off of the task was held in Bad Tölz, Germany, from February 11-13, 2009.

\section{THERMAL ENERGY STORAGE TECHNOLOGIES}

There are several ways to classify the technologies with which thermal energy can be stored. One of these is to use the underlying physical principle for storage. This leads to four main groups of storage: sensible heat, latent heat, sorption and thermochemical storage. The research stage of these technologies ranges from market mature to fundamental research, respectively. In the following section, these technologies will be briefly described, as well as their typical applications.

\section{Sensible heat}

With sensible heat storage, thermal energy is stored in the heat capacity of a substance, for instance water. When heat is supplied to the water its temperature will rise, hence the word sensible. The heat capacity of the material determines the specific amount of energy that can be stored. Because of its very high heat capacity, water is an ideal heat storage medium. Therefore, the vast majority of heat storage systems currently use water as a storage medium. Above $100^{\circ} \mathrm{C}$, other materials like thermal oil or concrete are more suitable as sensible storage medium.

The range of storage capacities covered by sensible systems is very large. For district heating and large buildings, heat and cold storage systems with watercarrying underground layers (aquifers) are used. Different layers, on depths ranging from tens to hundreds of meters, are used to store the relatively warm and cold water separately. Capacities of aquifer thermal energy storage systems range from tens of thousands to more than a million cubic meter water equivalent.

Another type of large-scale sensible heat storage uses water pits or large buffer tanks. In these systems, the temperature is higher than in aquifer storage systems, so thermal losses must be minimised with thermal insulation. In many cases, these systems are used to store solar heat for district heating systems. Most systems of this type can be found in Denmark, combined with central solar thermal systems, and in Germany, where they are also combined with decentralised solar thermal collectors.

At the building level, insulated water tanks are used to store heat. Typical storage volumes range from 100 litres for solar domestic hot water heating up to several thousands for domestic space heating.

At high temperature, sensible heat can be stored in thermal oils like silicone oil, or in solid materials like concrete. An example of the latter is concentrated solar power: during the daytime, high temperature heat from concentrating parabolic trough receivers is partly used in a steam driven power plant, and partly the heat is stored at temperatures of 400 to $500^{\circ} \mathrm{C}$ in large, well insulated blocks of concrete. By storing the heat, the power plant is able to generate power around the clock.

\section{Latent heat}

It takes energy to let a substance change its phase, for instance from solid to liquid or from liquid to gas. The heat necessary for this transformation is called latent heat, because the phase transition takes place at a fixed temperature, without resulting in a temperature increase. Storage using latent heat storage can be more compact than storage using sensible heat. For instance, the heat needed to melt $1 \mathrm{~kg}$ of ice is equal to the heat needed to heat water from $0^{\circ} \mathrm{C}$ to $80^{\circ} \mathrm{C}$.

Latent heat can be stored in many types of materials, called phase change materials or PCM. At low temperatures, latent heat storage is used in refrigeration or cooling systems, either to boost the maximum peak output of the systems or to enable the use of cheap, night time electricity for refrigeration or cooling at daytime. Water/ice is often used as PCM in such applications.

At room temperatures, PCM storage is used to increase the passive or active solar fraction of room heating. For some time now, several building products have been introduced to the market with an integrated latent heat storage material, like wood fibre board or gypsum board. With these products, the heat capacity of construction elements in a building may be increased, helping to dampen indoor temperature fluctuations, thereby increasing indoor thermal comfort.

Latent storage of solar thermal energy for hot tap water production is still in development. As the temperature range in this application is rather large, latent heat storage loses its relative advantage of a high latent heat. The effectiveness of PCM is further decreased by its low thermal conductivity. Both the increase of latent heat storage capacity and of thermal conductivity are the main topics of R\&D for this technology.

At higher temperatures, e.g. for concentrated solar power applications, molten salts are used as latent heat storage medium. Typically, mixtures of sodium nitrate and potassium nitrate are applied to store heat between $300^{\circ} \mathrm{C}$ 
and $400^{\circ} \mathrm{C}$. In these applications, new materials are being searched for, that enable storage at higher temperatures, as higher driving temperatures increase the CSP plant efficiency.

\section{Sorption}

A third class of thermal storage technologies is sorption technology. In sorption, a vapour is adsorbed by a liquid or a porous solid, and heat is released that can be used externally. The temperature at which this process takes place depends on the substances used and on the pressure of the system. When heat is fed into in the system, the vapour is driven out of the sorbent. Well known sorption materials are silica gel and zeolite: these materials can take up water vapour with a mass equal to several times their own mass.

Sorption technologies are used in thermally driven cooling machines. The amount of active sorption material in these applications is not that large, so material cost is less of an issue than in typical heat storage applications. The development activities for sorption thermal storage materials are therefore mainly aimed at finding materials that are sufficiently cost-effective.

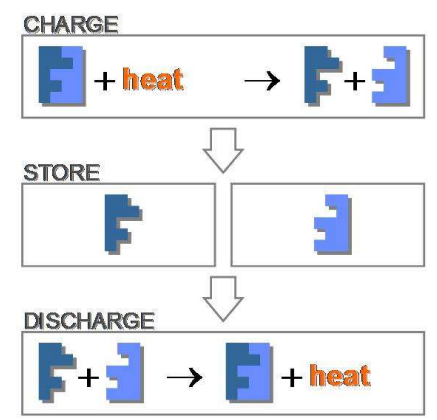

Figure 1 - Principle of thermochemical storage.

\section{Thermochemical}

The highest potential for compact thermal storage is in thermochemical materials. The development of this technology is still in an early stage. In thermochemical storage, heat is used to split a substance in two separate components, that can be stored separately without energy loss. When the heat is needed again, the two substances are brought together in a reactor, reproducing the original compound, and generating the required heat. In principle, every reversible reaction between two components can be used. Most chemical reactions, however, generate high temperatures and thus also need high temperatures for heat storage.

Most thermochemical materials that are currently under development are chemisorption materials. The storage principle is comparable to adsorption, but in this case the uptake of a vapour results in an actual change in the crystalline structure and the chemical properties of the material. Examples are salt hydrates, like copper sulphate, calcium chloride or magnesium sulphate. These have relatively low hydration temperatures, making them suitable candidates for the storage of solar thermal energy.

Other chemical reactions that are studied for the storage of higher temperatures (around $350^{\circ} \mathrm{C}$ ) are the reaction of magnesium oxide with water vapour to form magnesium hydroxide. Storage at even higher temperatures, between $450^{\circ} \mathrm{C}$ and $500^{\circ} \mathrm{C}$, is studied with the formation of ammonia from hydrogen and nitrogen.

\section{Storage densities of material classes $\left(\mathrm{MJ} / \mathrm{m}^{3}\right)$}

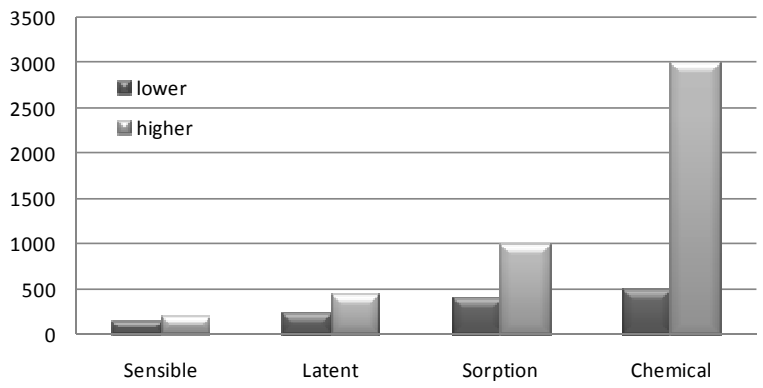

Figure 2 - Comparison of the range of storage densities for different thermal energy storage material classes.

\section{OBJECTIVE AND SCOPE}

The objective of the new IEA Task is to develop advanced materials for compact storage systems, suitable not only for solar thermal systems, but also for other renewable heating and cooling applications such as solar cooling, micro-cogeneration, biomass, heat pumps, concentrated solar power and district heating.

A secondary but equally important objective of this Task is to create an active and effective research network in which researchers and industry working in the field of thermal energy storage can collaborate.

The Task covers phase change materials, thermochemical and sorption materials, and composite materials and nanostructures. It includes activities such as material development, analysis, and engineering, numerical modelling of materials and systems, development of storage components and systems, and development of standards and test methods.

The main added value of this Task is to combine the knowledge of experts from materials science as well as solar/renewable heating and energy conservation. Already at the start of the Task, 1 January 2009, more than 60 experts from over 25 organisations from 15 countries started their contribution to the Task work. 
This work is subdivided into two main subtasks: Materials and Applications, reflecting the contribution from the two different IEA programs. Each subtask consists of several Working Groups that are briefly described below.

\section{Materials Engineering and Processing}

The heart of the work is in this working group, that has the objective to study and understand compact storage materials and develop new materials with better properties, and to develop new processing technologies that enable low-cost and large scale production of these materials. This is a long-term goal, which is not expected to be achieved within the duration of the Task. The work will include the synthesis of new materials, the determination of materials characteristics, finding optimal methods for micro- and macro encapsulation of storage materials and developing novel production principles.

The first activities in this Working Group include the characterisation of compound of salt hydrates as thermochemical materials, the improvement of synthesis methods of zeolites and the use of nano-sized particles for the stimulation of crystallisation in phase change materials.

\section{Materials Testing and Characterisation}

The performance characteristics of novel thermal energy storage materials like phase-change materials or thermochemical materials, often cannot be determined as straightforward as with sensible heat storage materials. In order to have proper comparison possibilities appropriate testing and characterisation procedures should be developed and assessed.

The activities in this Working Group are aimed at the development of these new procedures and include comparative testing of materials and their required methods, long-term stability determination and prestandardisation of testing methods.

\section{Numerical Modelling}

With highly sophisticated numerical models, it should be possible to find ways to optimize storage materials in combination with system components like a heat exchanger or a reactor. To this end, the existing models that describe the processes on very different length scales have to be combined. Using multiscale modelling, it is possible to make the right coupling between models on the molecular scale with models on the scale a grain of storage material or on the scale of a reactor.

Within this working group, steps are taken towards these multiscale models for phase change materials and thermochemical materials. The group has begun to describe the state-of-the-art modelling techniques for PCM and TCM on three different length scales: micro, meso and macro.

\section{Apparatus and Component Integration}

The storage apparatus is composed of the storage material and the equipment or components necessary to charge and discharge the storage material in a controlled and optimal way. These components include heat exchangers, pumps or fans, and chemical reactors. In order to arrive at optimised apparatus configurations, work will be done on storage container and reactor design, storage apparatus design based on the selected storage material, heat transfer optimisation and apparatus and components performance assessment.

\section{Applications}

The Applications subtask has three Working Groups, divided along the typical storage temperature of a group of applications: cooling, heating and domestic hot water, and high temperature applications. Although the applications themselves place very different requirements on storage technology, the steps that must be taken are very similar for all applications. Hence, the activities within the Working Groups in this Subtask are very similar as well.

The activities serve the underlying guidance principle of the materials development within the limitations of the application. The materials development will be directed by the desired system performance. A constant assessment of performance criteria for a given application will be used to determine the chances for a given material/system combination. These criteria can come from economic, environmental, production technology or market considerations. Activities in the Application Working Groups include, amongst others, definition of application boundary conditions, definition of required thermophysical properties for each application, selection of relevant candidate materials and system technologies and performance assessment and validation.

\section{Common Working Groups}

Theoretical Limits, System Integration and Dissemination are three working groups that are common and not part of a subtask. In the first, the theoretical limits of compact thermal storage materials and systems from a physical, technical and economical viewpoint are determined. An estimate is made of the maximum possible performance to be expected from a thermal storage system in a given application, giving a set of reference points for the comparison of lab tests, field tests and commercial systems.

In System Integration, a mapping is made of all the problems to be solved when making the storage system a part of the complete energy system.

Finally, within the Dissemination Working Group, the necessary growth of involvement of new experts, especially from the materials field, is assisted. 


\section{CONCLUSION}

The new Joint Task will help to make new compact thermal storage materials and components available in the future. These will enable a much higher fraction of solar thermal energy to be gained by solar thermal systems., and lead to better and more compact storage systems that will increase the efficiency of a number of other technologies, in buildings, industries, in districts and in power generation.

The work in this Task has started in the beginning of this year and will last 4 years. Although the group already exceeds 60 experts from 15 countries, a lot of work has to be done and certainly the hands and brains of more experts are needed to increase the chances of success. Hence, experts from industries and research organisations worldwide that are active in this field, are cordially invited to join the Task. If you are interested, please contact the authors of this paper or your national ECES or SHC ExCo member.

In the meantime, work on a political and decision-maker level has to be continued in order to increase the funding for better coordinated and more profound research in this field on an international level. Certainly, the creation of a new and strong Renewable Heating and Cooling European Technology Platform, in which Storage Technology is identified as a key technology, will help this process. But most important is to attract young and enthusiastic researchers that can help to make compact thermal storage a real enabling technology.

\section{MORE INFORMATION}

More information on this task can be found on the Task websites at www.iea-shc.org/task42 or on www.iea-eces.org.

\section{REFERENCES}

[1] www.fskab.com/annex17/

[2] www.iea-shc.org/task32/ 